\title{
Stillhäufigkeit und Stilldauer in Deutschland - eine systematische Übersicht
}

\author{
Breastfeeding Rates and Duration in Germany - A Systematic Review
}

Autoren

Institute
A. Weissenborn ${ }^{1}$, M. Abou-Dakn², R. Bergmann ${ }^{3}$, D. Both ${ }^{4}$, R. Gresens ${ }^{5}$, B. Hahn ${ }^{6}$, A. Hecker ${ }^{1}$, B. Koletzko ${ }^{7}$, M. Krawinkel ${ }^{8}$, D. Kroll ${ }^{9}$, E. Rouw ${ }^{10}$, M. Scheele ${ }^{11}$, U. Schwegler ${ }^{12}$, E. Sievers ${ }^{13}$, E. Sporleder ${ }^{14}$, S. Springer ${ }^{15}$, K. Vetter ${ }^{16}$, A. Wöckel ${ }^{17}$, M. Kersting ${ }^{18}$

Die Institutsangaben sind am Ende des Beitrags gelistet

\section{Schlüsselwörter \\ - Stillraten \\ - Stillbeginn \\ - Monitoring \\ - Stillförderung \\ - Frühe Hilfen}

\section{Key words \\ - breastfeeding \\ - monitoring \\ - infant feeding \\ - breastfeeding support}

Bibliografie

DOI http://dx.doi.org/

10.1055/s-0035-1555946

Online-Publikation: 3.9.2015

Gesundheitswesen 2016;

78: 695-707

(c) Georg Thieme Verlag KG

Stuttgart · New York

ISSN 0941-3790

Korrespondenzadresse

Dr. rer. medic. Anke

Weissenborn

Bundesinstitut für

Risikobewertung

Lebensmittelsicherheit

Max-Dohrn-Straße 8-10

10589 Berlin

anke.weissenborn@bfr.bund.de

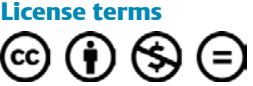

\section{Abstract}

$\nabla$

Ziel: Die vorliegende Arbeit hat zum Ziel, 20

Jahre nach Einrichtung der Nationalen Stillkommission anhand der in Deutschland publizierten Stilldaten einen Einblick in die Entwicklung des Stillverhaltens zu geben.

Methodik: Zur Identifizierung relevanter Publikationen wurde eine umfassende Literaturrecherche in den Datenbanken PubMed und Web of Science mit den Suchbegriffen „breast feeding" oder „breastfeeding“ in Kombination mit „Germany“ durchgeführt. Der Publikationszeitraum wurde dafür auf die Jahre 1995-2014 begrenzt.

Ergebnisse: Insgesamt wurden 35 Studien mit Daten zum Stillen für die Geburtsjahrgänge 1990-2012 erfasst. Die Daten stammen mehrheitlich aus regionalen oder lokalen Erhebungen und sind überwiegend retrospektiv erhoben worden. Etwa $60 \%$ der Studien waren mit dem primären Ziel durchgeführt worden, Daten zum Stillen bzw. zur Ernährung im Säuglingsalter zu erheben. Trotz hoher Stillraten zu Beginn (72-97\%) ist nach wie vor in den ersten 2 Monaten der stärkste Abfall der (Voll-)Stillraten zu verzeichnen, so dass bis zum Alter von 6 Monaten nur noch etwa $50 \%$ der Säuglinge überhaupt gestillt werden.

Schlussfolgerung: Verstärkte Stillfördermaßnahmen und unter anderem der Ausbau Früher Hilfen zum Stillen sind notwendig, um den Stillerfolg in Deutschland nachhaltig zu sichern. Mit Blick auf die Defizite bei der Stilldatenerhebung erscheint die Errichtung eines effizienten standardisierten Stillmonitorings in Deutschland unverzichtbar.

\section{Einleitung}

\section{$\nabla$}

Stillen ist für Mutter und Kind mit positiven gesundheitlichen Effekten assoziiert und trägt zur Förderung der emotionalen Bindung zwi-

\section{Abstract \\ $\nabla$}

Aim: 20 years after establishment of the National Breastfeeding Committee, the present work, based on published data on breastfeeding, is aimed at providing insight into the development of breastfeeding behaviour in Germany.

Methods: To identify relevant publications, a comprehensive literature search was conducted in PubMed and Web of Science using the search terms "breast feeding" or "breastfeeding" in combination with "Germany". The publication period was limited to the period 1995-2014.

Results: A total of 35 studies with data on breastfeeding for the birth cohorts of 1990-2012 were identified. Most of the data had been collected in regional or local surveys, often retrospectively. About $60 \%$ of the studies had been conducted with the primary aim of collecting data on breastfeeding or infant nutrition. Over the past 2 decades, breastfeeding rates were always relatively high at the beginning (72-97\%). However, they declined significantly within the first 2 months, and by the age of 6 months, only about $50 \%$ of infants were still breastfed.

Conclusion: Breastfeeding support and early assistance should be offered to a greater extent in order to achieve sustainable improvement of breastfeeding frequency and duration in Germany. Regarding the quality of data collected on breastfeeding, it seems crucial to implement standardised approaches to monitor breastfeeding in Germany. 
den. Eines der formulierten Ziele, bis zum Jahr 1995 in allen Ländern Stillkoordinatoren zu berufen und Stillkommissionen einzurichten, wurde in Deutschland 1994 durch die Einrichtung der Nationalen Stillkommission (NSK) umgesetzt [3]. Die Gründung der NSK erfolgte mit dem Ziel, das Stillen und die Entwicklung einer neuen Stillkultur in Deutschland zu fördern und dazu beizutragen, dass Stillen zur normalen Ernährung für Säuglinge wird. Dieses soll durch Beratung der Bundesregierung, Herausgabe von Richtlinien und Empfehlungen und Unterstützung von Initiativen zur Beseitigung von Stillhindernissen erreicht - und der Erfolg der Arbeit durch Datenerhebungen und Berichte dokumentiert werden [3]. Seit ihrer Gründung hat die NSK gemeinsam mit den in der Kommission vertretenen Berufsverbänden eine Reihe von Empfehlungen zu Themen rund um das Stillen herausgegeben (z.B. [4]). Außerdem spricht sie sich seit vielen Jahren für die Einrichtung eines Stillmonitorings aus und hat dafür im Jahr 2009 ein Konzept veröffentlicht [5]. Allerdings wurden bis heute in Deutschland keine systematischen Monitoring- und Evaluierungsstudien zur Bewertung der Stillförderung durchgeführt. Es gibt aber eine Reihe von Studien, in denen teilweise mit anderer Zielsetzung Stilldaten erhoben wurden. Ziel dieser Arbeit war es daher, 20 Jahre nach Einrichtung der NSK auf der Basis publizierter Stilldaten einen Einblick in die Entwicklung des Stillverhaltens in Deutschland zu geben.

\section{Methoden}

$\nabla$

Zur Identifizierung relevanter Publikationen wurde in den Datenbanken PubMed und Web of Science im Publikationszeitraum 1995-2014 eine umfassende Literaturrecherche mit den Suchbegriffen „breast feeding“ oder „breastfeeding“ in Kombination mit „Germany“ durchgeführt. Es wurden nur Studien einbezogen, in denen mit primärer oder sekundärer Zielsetzung Daten zum Stillen (Stillbeginn, Dauer und/oder Intensität des Stillens) erhoben worden waren. Studien, in denen lediglich Einstellungen oder Kenntnisse zum Stillen oder das Stillverhalten bei Frühgeborenen oder kranken Säuglingen untersucht wurden, blieben unberücksichtigt. Die Referenzlisten der identifizierten Publikationen wurden zudem nach weiteren potenziell relevanten Artikeln durchsucht. Da die genannten Datenbanken ausschließlich Publikationen in englischer Sprache enthalten, wurde zusätzlich eine Internetrecherche mit deutschen Suchbegriffen durchgeführt, um auch Berichte von Gesundheitsämtern oder anderen Institutionen des öffentlichen Gesundheitsdienstes sowie Abschlussarbeiten wie z. B. Master- oder Doktorarbeiten zu erfassen. Die Studiencharakteristika und die aus den Studien extrahierten Daten zum Stillen wurden tabellarisch zusammengestellt und die Gesamt- und Vollstillraten für die Altersstufen 2, 4 und 6 Monate zusätzlich grafisch dargestellt.

\section{Ergebnisse \\ $\nabla$}

\section{Studien zum Stillverhalten}

Durch Recherche in den beiden medizinischen/wissenschaftlichen Datenbanken wurden 638 Treffer erzielt, von denen nach Durchsicht der Titel und Abstracts 67 Publikationen im Volltext bestellt und schließlich 27 Publikationen in die Auswertung einbezogen wurden. Nach Durchsicht der Referenzlisten der Artikel und durch Recherche im Internet wurden 8 weitere Erhebungen identifiziert, sodass insgesamt 35 Publikationen mit Daten zum Stillen für die Geburtsjahrgänge 1990-2012 einbezogen wurden [6-8, 11-40]. Etwas mehr als die Hälfte der Studien (57\%) war mit dem primären Ziel durchgeführt worden, Daten zum Stillen bzw. zur Ernährung im Säuglingsalter zu erheben; in den anderen Studien war die Erhebung des Stillverhaltens mit anderen Zielsetzungen erfolgt. Die erfassten Daten stammen überwiegend aus regionalen oder lokalen Erhebungen. Vier bundesweite Erhebungen wurden identifiziert. Davon war eine prospektiv, von der Geburt bis zum Ende des ersten Lebensjahres [6], die anderen retrospektiv, im Rahmen des Kinder und Jugendgesundheitssurveys (KiGGS) $[28,29]$ sowie im Rahmen einer online-Verbraucherbefragung [25] durchgeführt worden. Nur in der DONALD-Studie (Dortmund Nutritional and Anthropometric Longitudinally Designed Study) werden systematisch jährlich neue Studienteilnehmer rekrutiert, bei denen unter anderem Stilldaten prospektiv erhoben werden, wodurch die Untersuchung längerfristiger Trends ermöglicht wird [30,31].

In der Mehrheit der identifizierten Studien ( $n=19 ; 54 \%)$ erfolgte die Datenerhebung retrospektiv, wobei die Erinnerungszeiträume nur in 3 Untersuchungen [19-21] über 4-6 bzw. 12 Monate zurückreichten; in 5 der Studien waren es bis $\mathrm{zu} 3$ Jahre $[20,25,37-39]$, in 9 zwischen 5 und 7 Jahren $[11,15,26,27,29,32$, $34,36,40]$ und in 2 weiteren $2-9$ bzw. 13 Jahre [28,33]. In 2 Querschnittsstudien $[8,18]$ sowie in sämtlichen Arbeiten mit prospektivem Design $(n=12 ; 34 \%)$ wurden 24-Stunden-Recalls zur Datenerhebung verwendet. In 2 Fällen fehlten Informationen zum Studiendesign, da die Publikationen nicht im Original auffindbar waren und die Daten daher einer Sekundärquelle entnommen wurden $[13,14]$. Nur 2 Erhebungen im Rahmen des KiGGS wurden mit repräsentativen Stichproben durchgeführt, aber mit retrospektiven Erhebungsmethoden [28,29]. Weiterhin wurde in 2 Publikationen explizit darauf hingewiesen, dass die Rekrutierung der Mutter-Kind-Paare in babyfreundlichen Krankenhäusern durchgeführt wurde $[19,20]$. In einem anderen Fall fand die Datenerhebung im Anschluss an eine Schulung des Personals von Geburtskliniken und von Nachsorgehebammen zum Thema Stillen/Stillmanagement statt [22].

In der Mehrzahl der Arbeiten wurde zwischen ausschließlichem und/oder vollem und jeglichem Stillen differenziert. Die Differenzierung erfolgte jedoch nicht immer entsprechend den Definitionen von Weltgesundheitsorganisation (WHO) und NSK $[9,10]$ : ausschließliches Stillen=Säugling erhält nur Muttermilch und keine anderen Flüssigkeiten oder Nahrung (nur Tropfen oder med. Sirup); überwiegendes Stillen=Säugling erhält Muttermilch und nicht nahrhafte Flüssigkeiten wie Wasser oder Tee; volles Stillen =Summe aus ausschließlichem und überwiegendem Stillen. Da zudem bekannt ist, dass die Erfragung der Stillintensität mit Unsicherheiten behaftet sein kann [41-43], wurden Daten zum ausschließlichen Stillen in jedem Fall als „Volles Stillen“ gewertet und differenzierte Angaben zu ausschließlichem und überwiegendem Stillen zu „Vollem Stillen“ zusammengefasst ( $\bullet$ Tab. 1; $\bullet$ Abb. 1-3).

\section{Daten zum Stillverhalten}

Die vorliegenden Daten zeigen, dass von den 1990-2012 geborenen Säuglingen zwischen 72 und $97 \%$ nach der Geburt gestillt wurden, wobei die Daten in Abhängigkeit von der Studienregion und -methode, nicht jedoch vom Geburts- oder Erhebungsjahr, variieren ( $\bullet$ Tab. 1). Im Alter von 2 Monaten wurden in den 1990er Jahren je nach Studie zwischen 45 und $88 \%$ und in den 2000er Jahren zwischen 62 und $88 \%$ der Säuglinge gestillt 


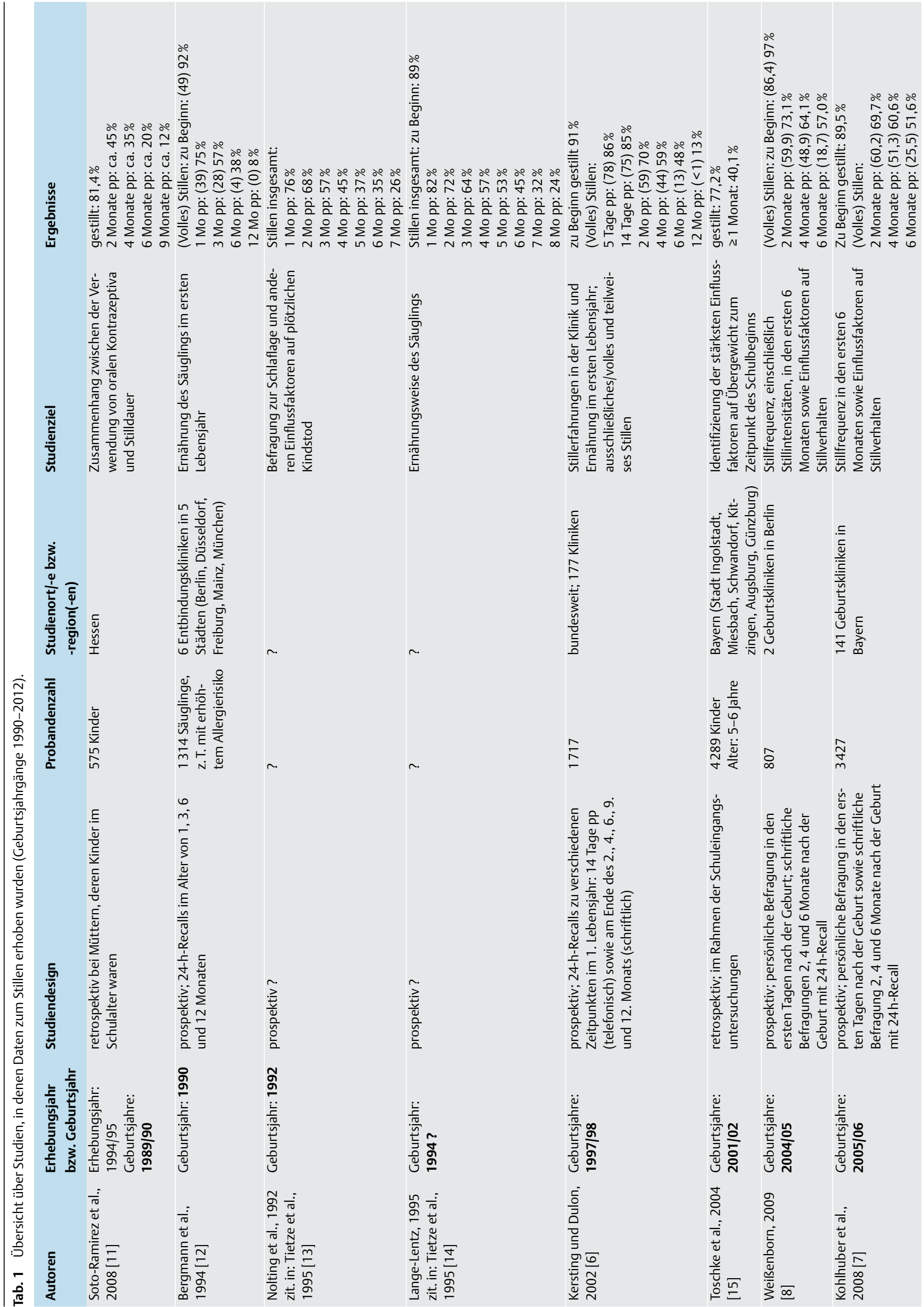




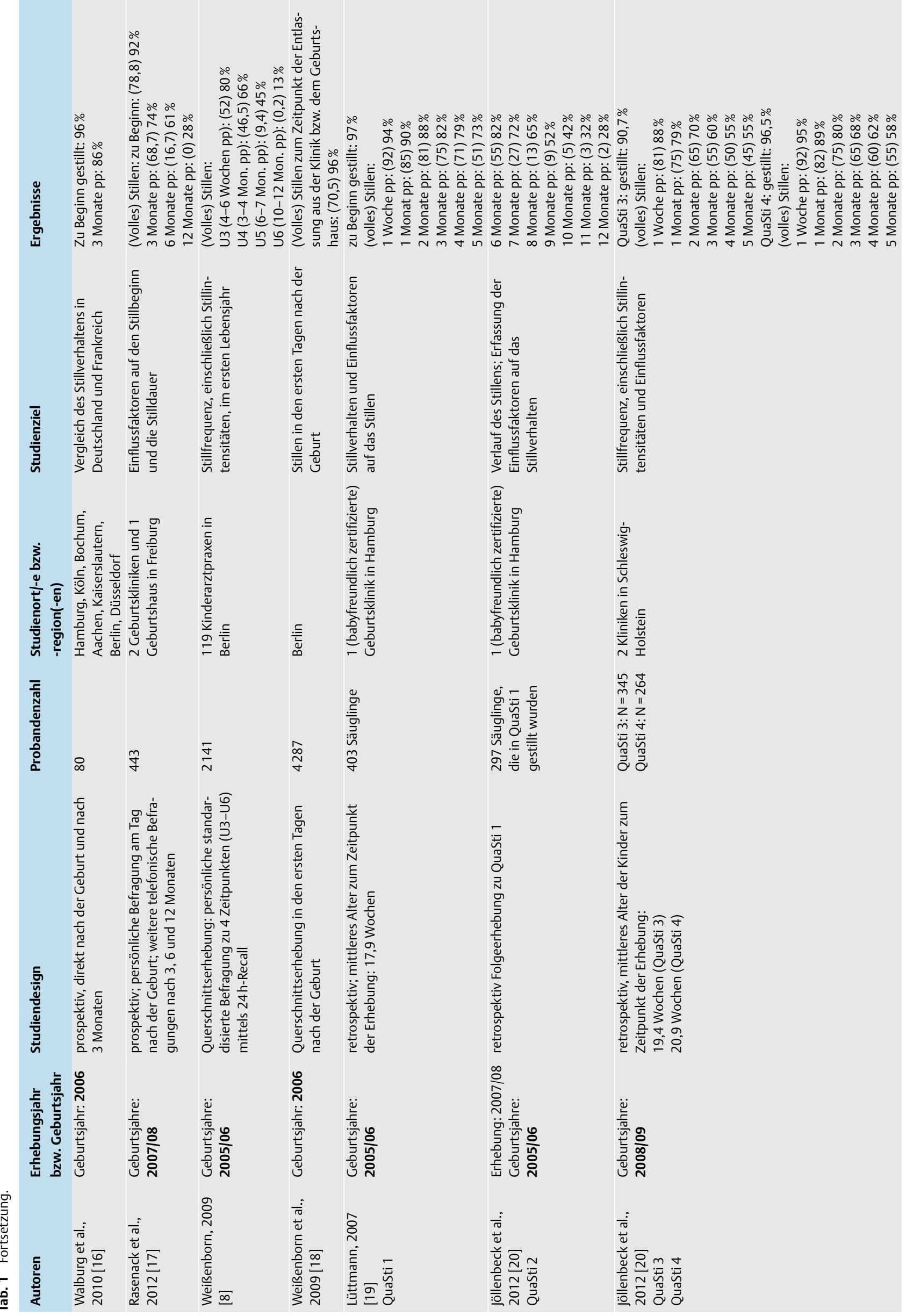




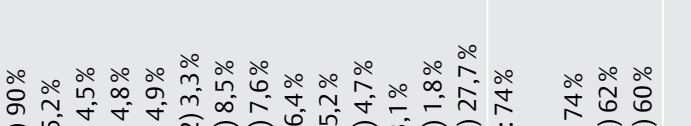

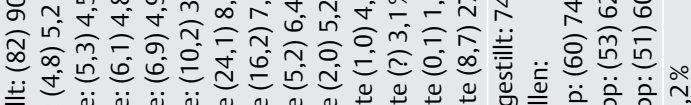

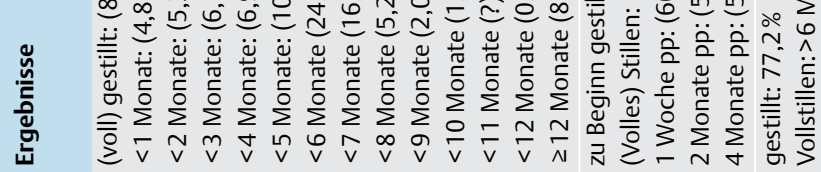
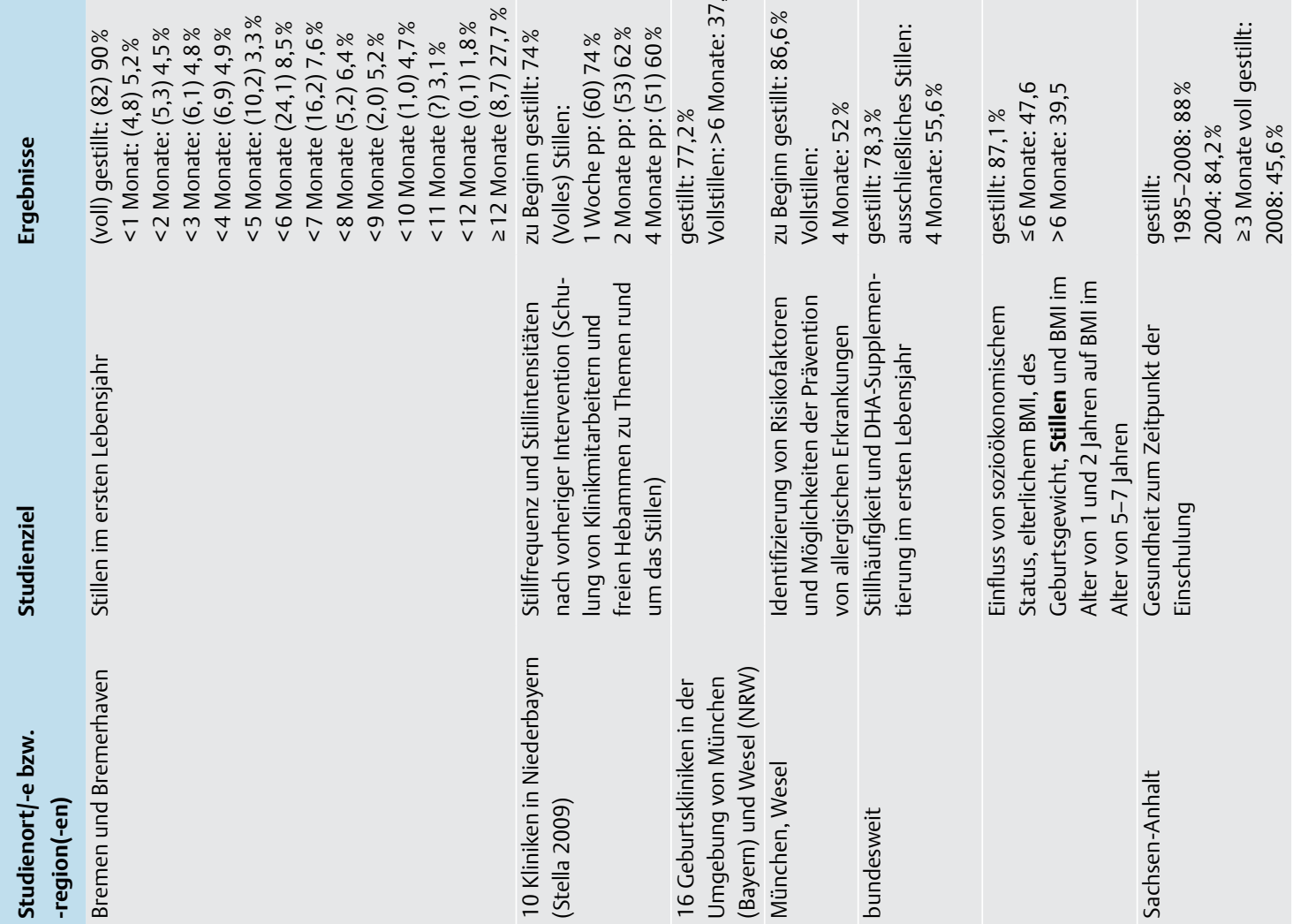

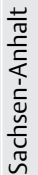

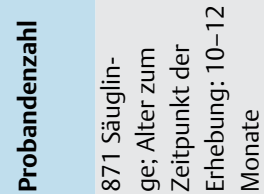

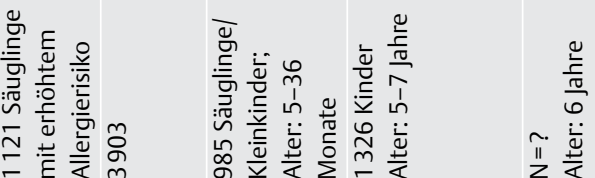

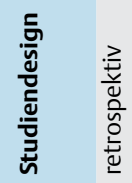

¿

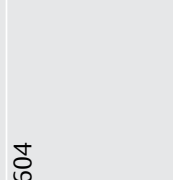

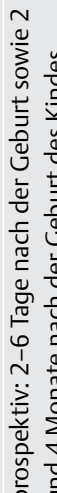
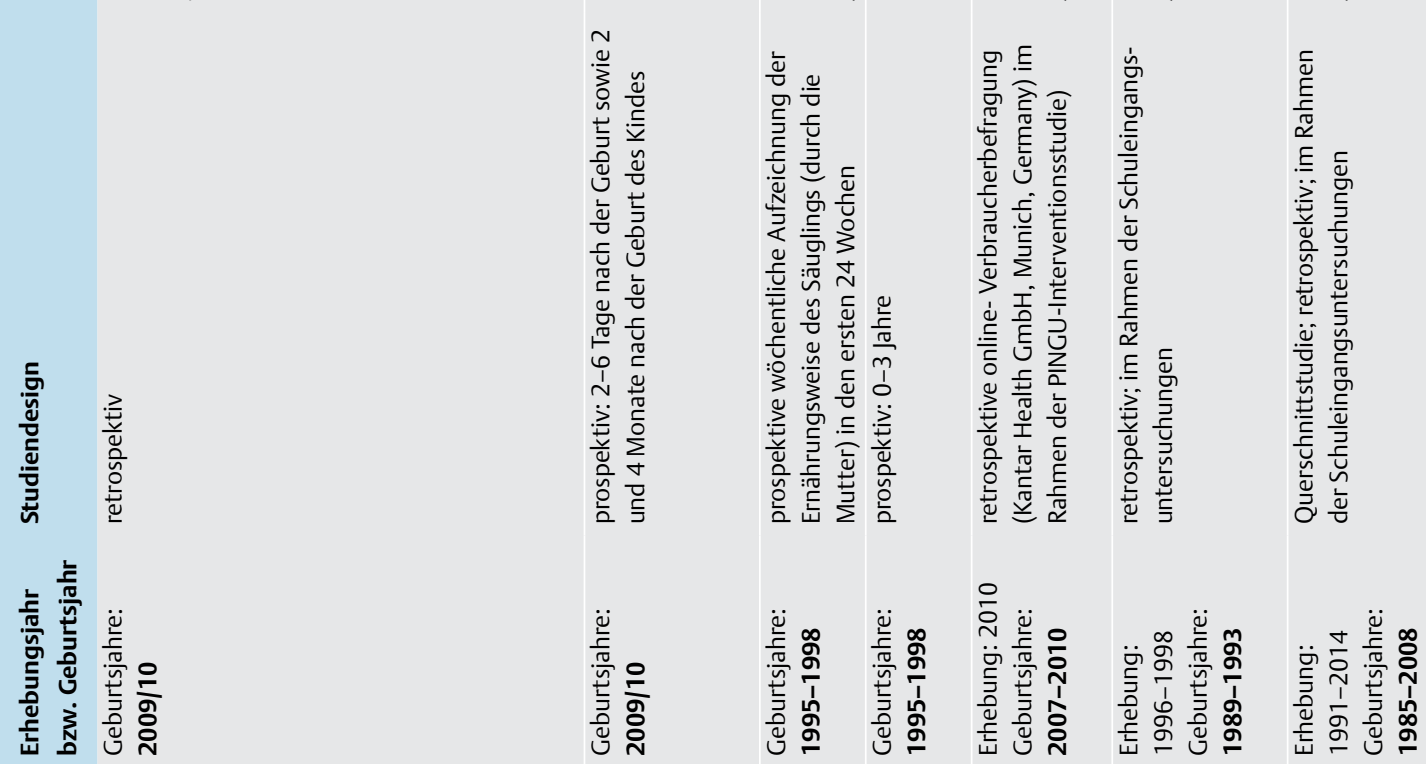

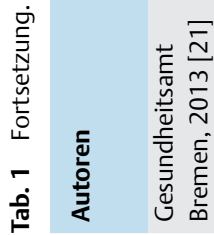

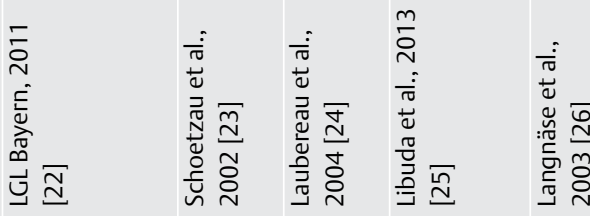

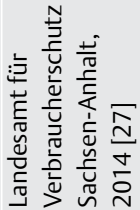




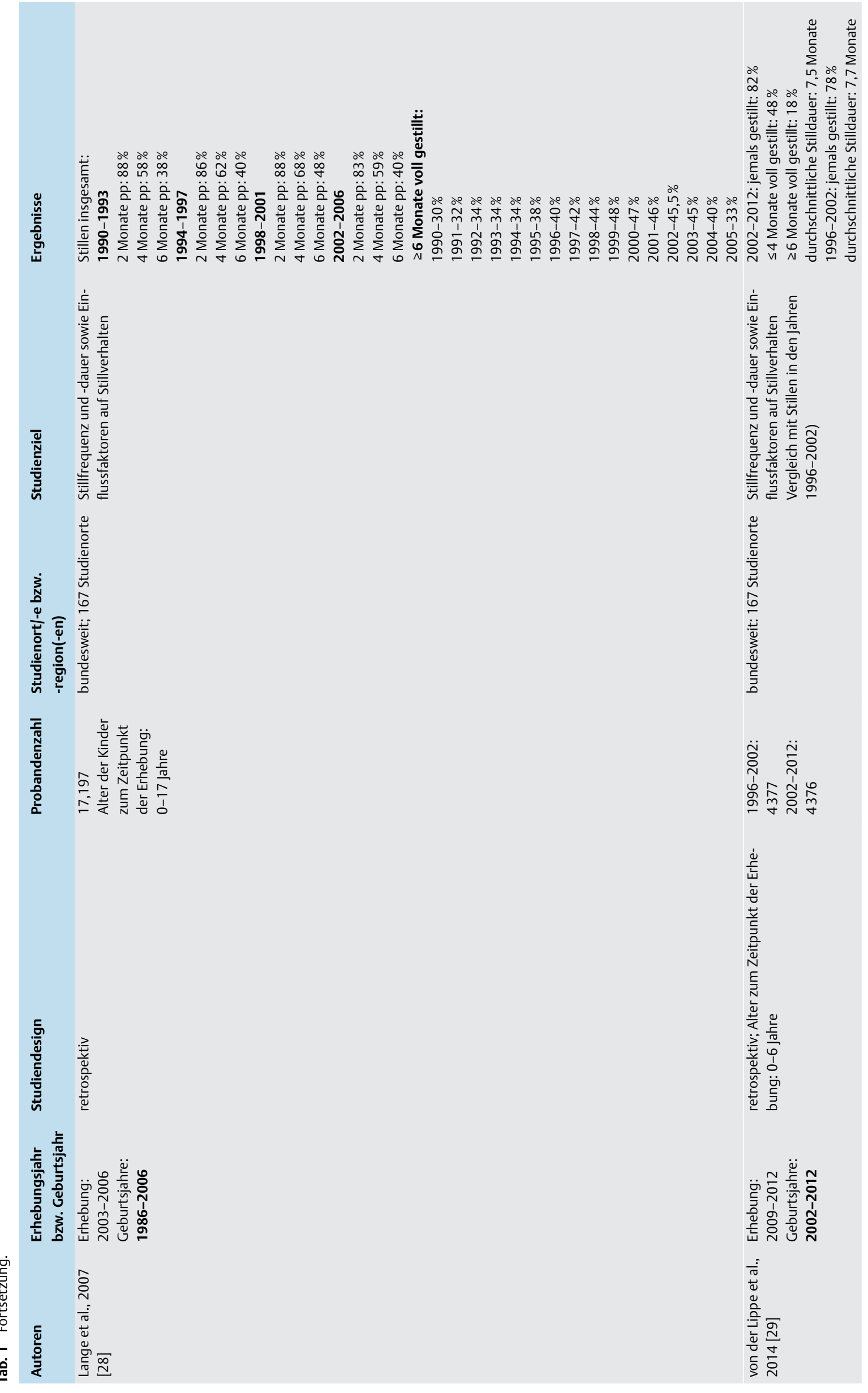



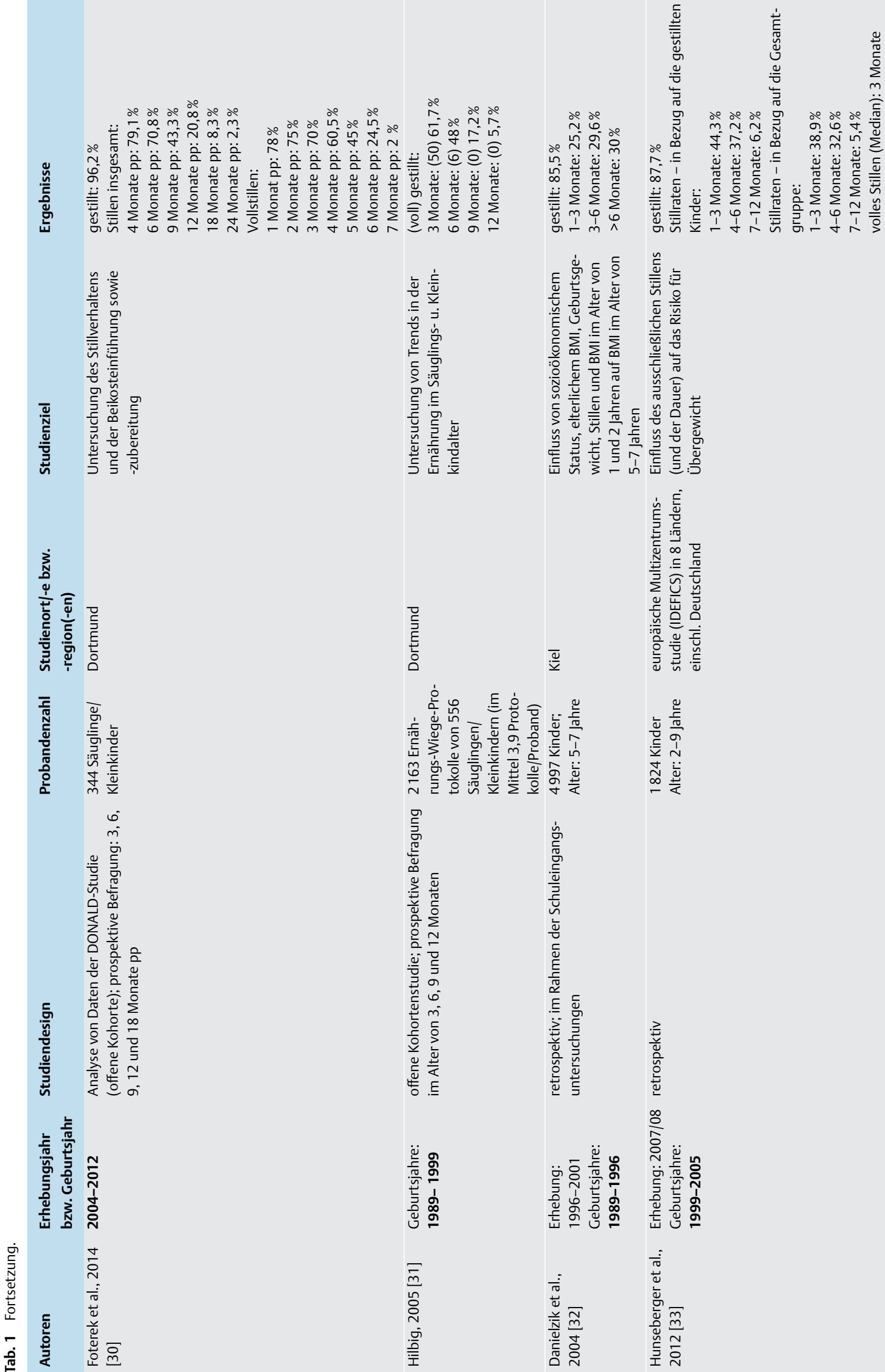

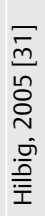

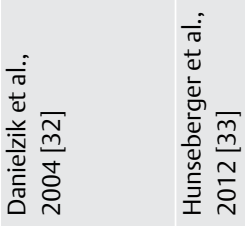




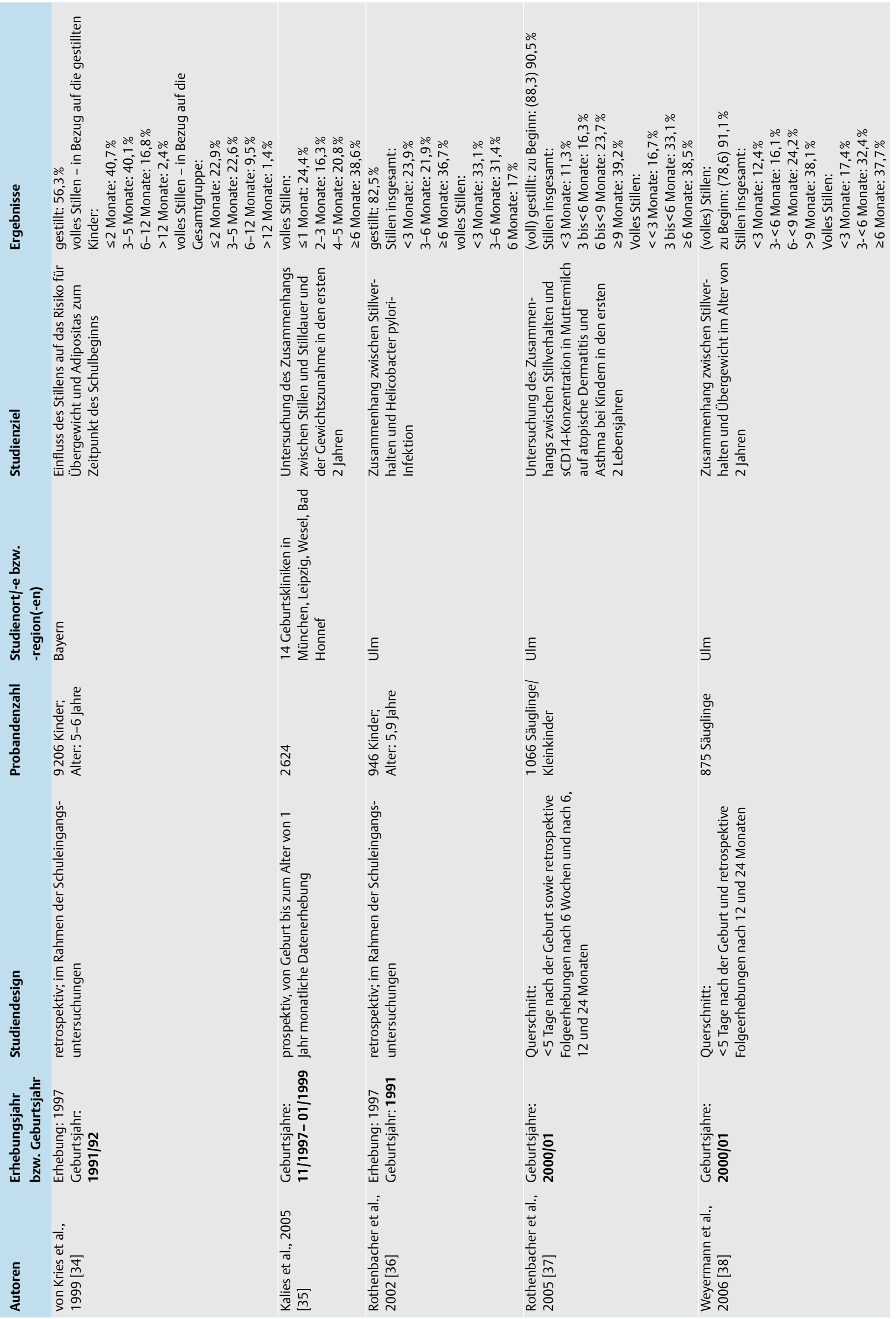




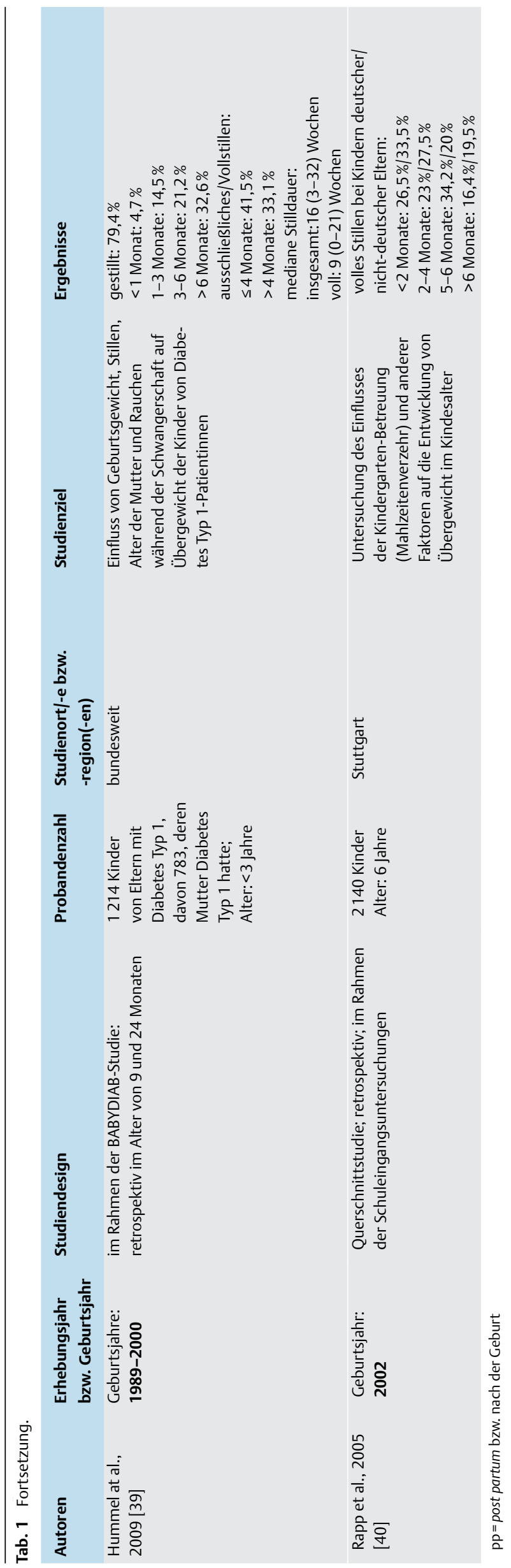

( $\bullet$ Abb. 1). Daten über volles Stillen im Alter von 2 Monaten liegen für die 1990er Jahre nur aus der SuSe-Studie vor, in der $1997 / 98$ eine Rate von 59\% ermittelt wurde [6]. In den 2000er Jahren wurden im Alter von 2 Monaten Vollstillraten zwischen 53 und $81 \%$ ermittelt ( $\bullet$ Abb. 1 ).

Im Alter von 4 Monaten wurden je nach Studie zwischen 35 und $79 \%$ der Säuglinge gestillt ( $\bullet$ Abb. 2). Daten zum vollen Stillen wurden für diese Altersstufe erstmals im Rahmen der SuSe-Studie 1997/98 erhoben; damals lag die Rate bei $44 \%$ [6]. In den 2000er Jahren lagen die Vollstillraten zwischen 46,5 und 71\%, wobei die niedrigste und die höchste Rate jeweils für denselben Geburtsjahrgang (2005/06) ermittelt wurde - in Berlin im Rahmen der Früherkennungsuntersuchungen in einer unselektierten Studiengruppe (46,5\%) [8] und in Hamburg (71\%) bei MutterKind-Paaren, die in einer babyfreundlichen Klinik entbunden und rekrutiert worden waren [19] ( $\bullet$ Abb. 2).

Im Alter von 6 Monaten wurden bei den in den 1990er Jahren geborenen Säuglingen Gesamt- und Vollstillraten zwischen 20 und $54 \%$ bzw. zwischen 11 und $48 \%$ ermittelt und bei den in den 2000er Jahren geborenen zwischen 40 und 82\% bzw. zwischen 17 und $55 \%$ ( $\odot$ Abb. 3, 4).

Aus den vorliegenden Daten lassen sich in keiner Altersgruppe eindeutige Veränderungen der Gesamt- oder Vollstillraten über die Zeit erkennen. Einzig in der DONALD Studie wurden im Zeitraum 1990-2013 signifikant positive Zeittrends für jegliches Stillen im Alter von 3 und 6 Monaten nachgewiesen. Für volles Stillen waren nur im Zeitraum 1990-2001 positive Trends zu sehen, im Zeitraum 2002-2013 stagnierten die Stillraten im Alter von 3 Monaten und waren im Alter von 6 Monaten tendenziell rückläufig (Libuda et al., under review).

\section{Diskussion}

In den vergangenen 2 Jahrzehnten wurde in Deutschland eine Vielzahl von Studien durchgeführt, in denen mit primärer oder sekundärer Zielsetzung Daten zum Stillen erhoben wurden. Somit besteht grundsätzlich Interesse an Untersuchungen zum Stillverhalten. Jedoch mangelt es an einer Standardisierung der Erhebungsmethoden, so dass die Daten nur schwer vergleichbar sind. Es lässt sich daher nicht zuverlässig beurteilen, ob fehlende Anzeichen für einen Anstieg der Stillraten real oder methodisch bedingt sind. So wurden in den meisten retrospektiven $[11,15$, $25,26,32-34,36,39]$ im Vergleich zu den zuletzt häufigeren prospektiven Erhebungen [6-8,12,16,17,24,30] niedrigere initiale Stillraten ermittelt. Dies kann zum einen mit den in prospektiven Studien meist strengen Einschlusskriterien (ausschließlich gesunde, reif geborene und normalgewichtige Säuglinge) erklärt werden. Zum anderen ist der Anteil nicht gestillter Säuglinge in prospektiven Studien oft bereits zu Beginn niedriger, weil diejenigen, die nicht oder nur kurze Zeit stillen (wollen), schwerer zur Teilnahme zu motivieren sind. Bei den Vollstillraten liegen die prospektiv erhobenen Raten niedriger als die retrospektiv erhobenen. Dies könnte darauf zurückzuführen sein, dass die Stillintensität (insbesondere das ausschließliche Stillen) in retrospektiven Erhebungen häufig überschätzt wird, während es selbst nach Jahren noch möglich ist, relativ zuverlässige Angaben über die Zahl der ,jemals gestillten“ Säuglinge und die Gesamtstilldauer zu erheben [41-45].

Eine weitere Erklärung für die beobachteten Unterschiede bei den für dieselben Geburtsjahrgänge erhobenen Daten sind ungleiche Rahmenbedingungen bei der Studiendurchführung, 


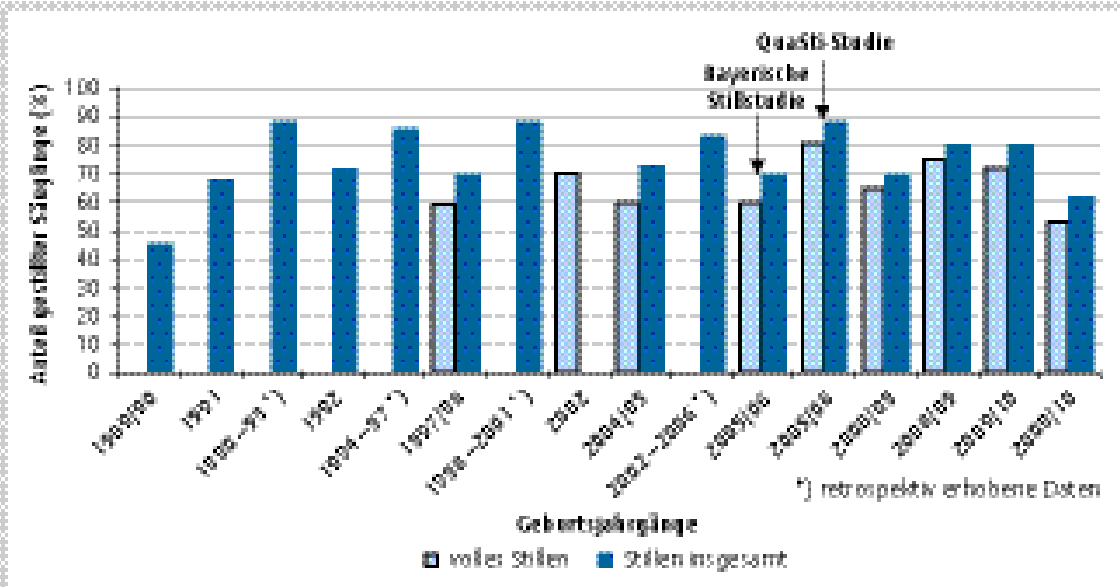

Abb. 1 Stillraten im Alter von 2 Monaten (Geburtsjahrgänge 1989/90-2009/10).

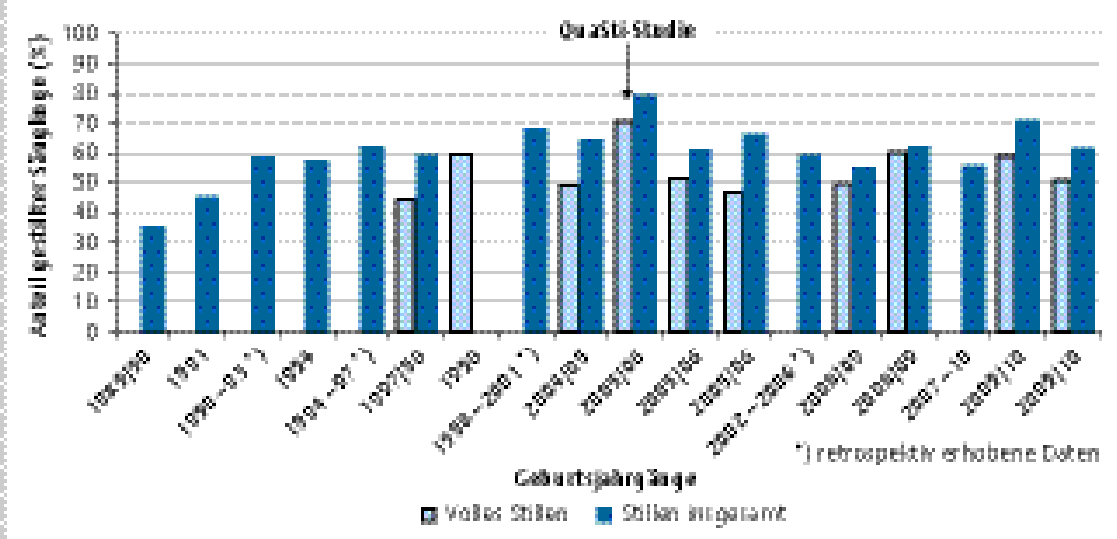

Abb. 2 Stillraten im Alter von 4 Monaten (Geburtsjahrgänge 1989/90-2009/10).

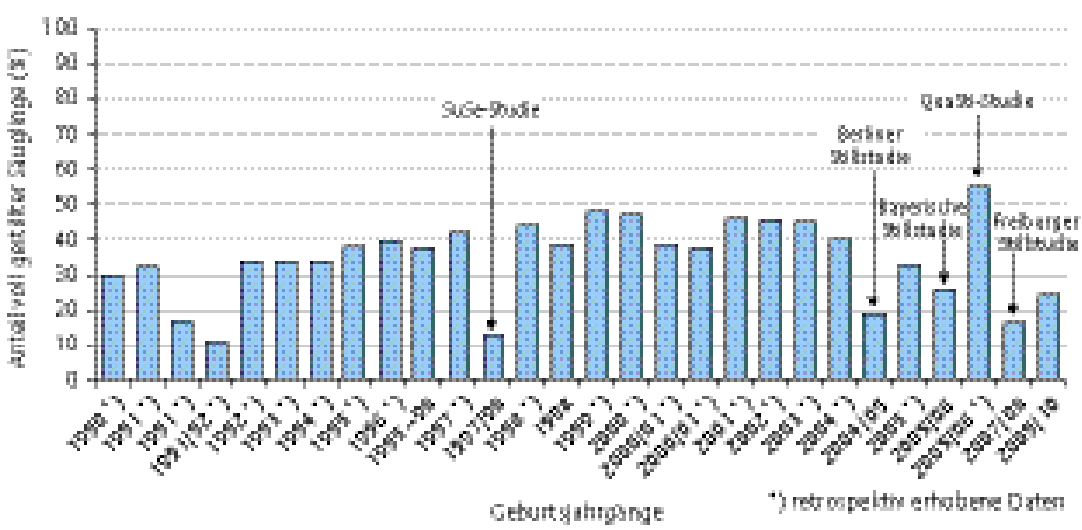

Abb. 3 Volles Stillen im Alter von 6 Monaten (Geburtsjahrgänge 1990-2009/10).

wie zum Beispiel regionale und soziale bzw. soziokulturelle Besonderheiten. Dies zeigte sich z.B. in Bayern, wo im Rahmen der bayerischen Stillstudie 2005/06 insgesamt etwa 90\% der Wöchnerinnen mit dem Stillen begonnen hatten, während in Niederbayern zur selben Zeit sowie auch in der so genannten STELLA-Studie im Jahr 2009/10 nur 74\% der Säuglinge zu Beginn gestillt wurden [7,22]. Die aktuellere STELLA-Studie in Niederbayern war dabei sogar im Anschluss an Schulungen von Klinikpersonal zum Stillen durchgeführt worden [46]. Somit lässt sich feststellen, dass die Personalschulungen in den Kliniken keinen direkten Effekt auf die initialen Stillraten hatten. Allerdings muss einschränkend gesagt werden, dass in der Studienregion von 2005/06 bis 2009/10 mehr Kaiserschnitte und kürzere Klinikaufenthalte nach der Geburt zu verzeichnen waren (U. Schwegler, pers. Mitteilung), die sich zusätzlich negativ auf die initialen
Stillraten ausgewirkt haben könnten. Ein anderes Beispiel für einen Einfluss der Rahmenbedingungen auf die ermittelten Stillraten ist die so genannte QuaSti-Studie, in der insbesondere im Alter von 4 und 6 Monaten im Vergleich zu anderen Studien die höchsten (Voll-)Stillraten erreicht wurden [19,20]. Dies ist vermutlich darauf zurückzuführen, dass die hier untersuchten Mutter-Kind-Paare in einer als babyfreundlich zertifizierten Hamburger Klinik rekrutiert und betreut wurden.

Interessant ist, dass etwa ein Drittel der vorliegenden Stilldaten im Rahmen von Schuleingangsuntersuchungen erhoben worden waren. Dabei lagen die Stillzeiten mindestens 5 Jahre zurück. Da die Schuleingangsuntersuchungen jedoch in Deutschland etabliert sind und regelmäßig durchgeführt werden, wären sie ohne großen Aufwand für ein Monitoring des Stillverhaltens nutzbar. 


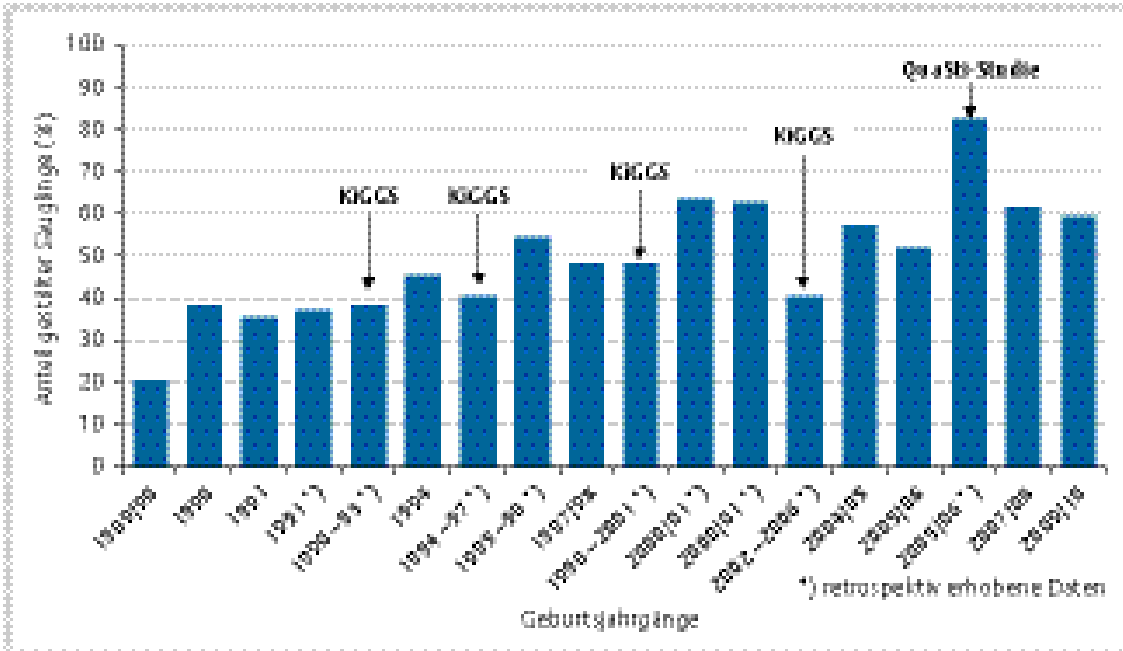

Abb. 4 Jegliches Stillen im Alter von 6 Monaten (Geburtsjahrgänge 1989/90-2009/10).

Trotz der eingeschränkten Vergleichbarkeit bestätigen die verfügbaren Daten den Eindruck aus bisherigen prospektiven Studien $[6-8,17]$, wonach trotz hoher initialer Stillraten innerhalb der ersten 2 Monate der stärkste Abfall der (Voll-)Stillraten zu verzeichnen ist und bis zum Alter von 6 Monaten nur noch etwa $50 \%$ der Säuglinge insgesamt gestillt werden. Anhand der Daten lassen sich weder für das Stillen zu Beginn noch für die betrachteten Altersstufen zeitliche Verläufe erkennen. Lediglich im Alter von 6 Monaten scheint sich ein leicht positiver Trend in der Entwicklung der Gesamtstillraten anzudeuten. Da jedoch für diese Altersstufe nur relativ wenige Daten zum Gesamtstillen vorliegen, bleibt abzuwarten, ob dieser Trend durch weitere Studien bestätigt wird. Auffallend viele Daten liegen zum vollen Stillen im Alter von 6 Monaten vor. Allerdings variieren diese so stark wie in sonst keiner Altersgruppe, so dass sich auch aus diesen Daten kein eindeutiger Trend ablesen lässt. Nimmt man die Daten der DONALD Studie mit der gebotenen Vorsicht aufgrund des lokalen Charakters und des überdurchschnittlichen formalen Bildungsniveaus der Mütter hilfsweise als ,Monitor', dann zeichnet sich in den letzten 10 Jahren für das volle Stillen eine Stagnation, wenn nicht sogar ein abnehmender Trend ab. Wenn Mütter aus höheren sozialen Schichten beim Stillen als Trendsetter wirken, stimmen diese Ergebnisse bedenklich. Sie belegen auch die Bedeutung und Notwendigkeit der im Europäischen Aktionsplan zum Stillen formulierten Maßnahmen zur Stillförderung für Deutschland. Dazu zählen z.B. die Sicherstellung einer sachkundigen Betreuung und Anleitung von Müttern zum Stillen, der Ausbau Früher Hilfen, die verstärkte Unterstützung von berufstätigen Müttern und deren Familien sowie die Verbesserung der Aus-, Weiter- und Fortbildung von Fachpersonal zum Stillen [47].

\section{Schlussfolgerung \\ $\nabla$}

Zusammenfassend deuten die verfügbaren Daten darauf hin, dass seit Beginn der 1990er Jahre relativ konstant zwischen 70 und etwa $90 \%$ der Mütter in Deutschland zu stillen beginnen, aber nach wie vor ein rapider Abfall der Stillraten innerhalb der ersten 2 Monate zu verzeichnen ist, so dass nur etwa $50 \%$ der Säuglinge mit 6 Monaten noch gestillt werden. Anhand der über 2 Jahrzehnte ermittelten (Voll-)Stillraten lässt sich kein klarer zeitlicher Trend ablesen. In lokalen Längsschnittdaten deutet sich allerdings eine Stagnation oder sogar ein abnehmender
Trend beim vollen Stillen in den letzten 10 Jahren an. Die bisherige Arbeit der NSK und die Stillförderung durch einschlägige (Berufs-)Gruppen in Deutschland scheinen somit keinen messbaren Effekt auf das Stillverhalten gehabt zu haben. Daher sind in Deutschland künftig verstärkt Stillfördermaßnahmen, auch unter Berücksichtigung von besonders bedürftigen Gruppen, notwendig.

Einschränkend muss angemerkt werden, dass angesichts der dargestellten heterogenen Erhebungsbedingungen auf der Basis der verfügbaren Daten keine zuverlässige Einschätzung der Entwicklung des Stillverhaltens möglich ist. Auch in anderen europäischen Ländern fehlt es zum Teil an systematisch erhobenen Stilldaten $[47,48]$. Daher war eine der Schlussfolgerungen aus dem EU-Projekt zur „Förderung des Stillens in Europa”, dass ein EU-weites Monitoring zur Erhebung von standardisierten Daten über das Stillverhalten dringend erforderlich ist, wobei die Verantwortung dafür bei den europäischen Mitgliedsstaaten liegt [47]. Mit Blick auf die dargestellten Defizite erscheint die Errichtung eines effizienten standardisierten Stillmonitorings in Deutschland unverzichtbar. Wie in dem 2009 von der NSK veröffentlichten Konzept dargelegt, könnten in Geburtskliniken und -häusern systematische, flächendeckende Datenerhebungen über den Stillbeginn durchgeführt werden, während das Stillverhalten im ersten Lebensjahr prospektiv im Rahmen der Kinderfrüherkennungsuntersuchungen oder retrospektiv im Rahmen der Schuleingangsuntersuchungen erhoben werden könnte. Dazu müssten die Stilldatenerhebungen fester Bestandteil dieser Untersuchungen werden und eine zentrale Datenverarbeitung und -auswertung sichergestellt werden. Darüber hinaus sollten in regelmäßigen Abständen qualitative Datenerhebungen zur Ermittlung von Einflussfaktoren auf das Stillen durchgeführt werden, damit die Wirksamkeit von Stillfördermaßnahmen zuverlässig bewertet und auf Misserfolge mit gezielten Maßnahmen reagiert werden kann $[5,8]$.

Interessenkonflikt: Die Autoren Abou-Dakn, Bergmann, Both, Gresens, Hahn, Kersting, Koletzko, Krawinkel, Kroll, Rouw, Scheele, Schwegler, Sievers, Sporleder, Springer, Vetter und Wöckel sind oder waren in den letzten 3 Jahren Mitglieder der Nationalen Stillkommission und sind teilweise Mitglieder in Verbänden, die Stillförderung als Ziel haben (siehe Auflistung unter Affiliations/Institute). 
Institute

${ }^{1}$ Bundesinstitut für Risikobewertung, Lebensmittelsicherheit, Berlin

${ }^{2}$ Klinik für Gynäkologie und Geburtshilfe, St. Joseph-Krankenhaus, Berlin

${ }^{3}$ Zentrum für Frauen, Kinder- und Jugendmedizin mit Perinatalzentrum,

Charité Universitätsmedizin Berlin, Berlin

${ }^{4}$ La Leche Liga e. V., Deutschland, Füssen-Weißensee

${ }^{5}$ Deutscher Hebammenverband e. V., Deutschland, Hamburg

${ }^{6}$ Berufsverband Kinderkrankenpflege Deutschland e. V., Deutschland, Neuss

${ }^{7}$ Dr. von Haunersches Kinderspital, Ludwig-Maximilians-Universität Mün-

chen, München

${ }^{8}$ Institut für Ernährungswissenschaft, Justus-Liebig-Universität, Giessen

${ }^{9}$ Berufsverband der Kinder- und Jugendärzte e.V., Deutschland, Berlin

${ }^{10}$ Arbeitsgemeinschaft freier Stillgruppen e.V., Deutschland, Bühl

${ }^{11}$ Bundesverband der Frauenärzte e.V., Deutschland, Hamburg

${ }^{12}$ LGL, Deutschland

${ }^{13}$ Akademie für öffentliches Gesundheitswesen, Sozialpädiatrie und KJGD,

Düsseldorf

${ }^{14}$ Verband Europäischer Laktationsberaterinnen e. V., Deutschland, Hannover

${ }^{5}$ Deutsche Gesellschaft für Sozialpädiatrie und Jugendmedizin e.V.,

Deutschland, Leipzig

${ }^{16}$ AG Hebammenwissenschaft, Medizinische Hochschule Hannover, Hannover

${ }^{17}$ Universitätsklinikum Ulm, Universitäts-Brustzentrum Ulm, Frauenklinik, Ulm

${ }^{18}$ Forschungsinstitut für Kinderernährung, Dortmund

\section{References}

1 Ip S, Chung M, Raman $G$ et al. Breast-feeding and Maternal Health Outcomes in Developed Countries. Evidence Report/Technology Assessment No. 153. Rockville, MD: Agency for Healthcare Research and Quality; 2007 (AHRQ Publication No. 07-E007)

2 Koletzko B, Bauer CP, Brönstrup A et al. Säuglingsernährung und Ernährung der stillenden Mutter. Aktualisierte Handlungsempfehlungen des Netzwerks Gesund ins Leben - Netzwerk Junge Familie, ein Projekt von IN FORM. Monatsschr Kinderheilkd 2013; 161: 237-246

3 Tietze KW, Truhmann B, Sedemund C., Hrsg. Stillen in Deutschland. Robert Koch-Institut; 1995: 7

4 Nationale Stillkommission am BfR. Empfehlungen zur Stilldauer. Empfehlungen der Nationalen Stillkommission vom 1. März 2004. Bundesgesundheitsblatt Gesundheitsforschung Gesundheitsschutz 2004; 47: 908

5 Nationale Stillkommission am BfR. Still-Monitoring in Deutschland. Konzept der Nationalen Stillkommission vom 21.12.2009 http://www. bfr.bund.de/cm/343/still_monitoring_in_deutschland_konzept.pdf

6 Kersting $M$, Dulon M. Fakten zum Stillen in Deutschland. Ergebnisse der SuSe-Studie. Monatsschr Kinderheilkd 2002; 150: 1196-1201

7 Kohlhuber M, Rebhan B, Schwegler $U$ et al. Breastfeeding rates and duration in Germany: a Bavarian cohort study. Br J Nutr 2008; 99: 1127-1132

8 Weißenborn A. Drei Studien über das Stillverhalten von Berliner Müttern als Beitrag zur Einrichtung eines Stillmonitorings in Deutschland. Dissertation an der Medizinischen Fakultät Charité - Universitätsmedizin Berlin 2009

9 Nationale Stillkommission am BfR. Einheitliche Terminologie zur Säuglingsernährung.Empfehlung der Nationalen Stillkommission von 1999; aktualisiert 2007; http://www.bfr.bund.de/cm/207/einheitliche_terminologie_zur_saeuglingsernaehrung.pdf

$10 \mathrm{WHO}$ (World Health Organization). Indicators for assessing infant and young child feeding practices.Conclusions of a consensus meeting held 6-8 November 2007 in Washington DC, USA Geneva, Switzerland: World Health Organisation; 2008; http://whqlibdoc.who.int/publica tions/2008/9789241596664_eng.pdf

11 Soto-Ramírez $N$, Karmaus $W$. The use of oral contraceptive before pregnancy and breast-feeding duration: a cross-sectional study with retrospective ascertainment. Int Breastfeed J 2008; 3: 29

12 Bergmann RL, Dudenhausen J, Bergmann E et al. Wie werden Säuglinge in Deutschland ernährt? Monatsschr Kinderheilkd 1994; 142: 412-417

13 Nolting HD, Schlegelmilch S, Truman B et al. Schlaflagen, Schlafumgebung und Schlafverhalten von Säuglingen. In: Tietze KW, Truhmann B, Sedemund C, Hrsg. Stillen in Deutschland. Robert Koch-Institut; 1995: 33-38

14 Lange-Lentz B. Zufriedenheit und Ernährungsweise des Säuglings. In: Tietze KW, Truhmann B, Sedemund C, Hrsg. Stillen in Deutschland. Robert Koch-Institut; 1995: 33-38

15 Toschke AM, Beyerlein A, von Kries R. Children at high risk for overweight: a classification and regression trees analysis approach. Obes Res 2005; 13: 1270-1274

16 Walburg V, Goehlich $M$, Conquet $M$ et al. Breast feeding initiation and duration: comparison of French and German mothers. Midwifery 2010; 26: 109-115
17 Rasenack R, Schneider C, Jahnz E et al. Einflussfaktoren auf die Stilldauer im Freiburger Geburtenkollektiv (FreiStill). Geburtsh Frauenheilk 2012; 72: $1-7$

18 Weissenborn A, Martin A, Bergmann R et al. Survey on breast-feeding initiation and potential impact factors in hospitals and birth centres in Berlin. Gesundheitswesen 2009; 71: 332-338

19 Lüttmann U. Ein Konzept für integrierte Stillförderung auf der Grundlage von Ergebnissen einer Stillstudie im „Babyfreundlichen Krankenhaus“. Diplomarbeit. Hochschule für Angewandte Wissenschaften (HAW) Hamburg 2007; http://edoc.sub.uni-hamburg.de/haw/volltexte/2008/555/pdf/ges_y_132.pdf

20 Jöllenbeck $M$. Identifikation wirksamer Interventionsmaßnahmen zur Stillförderung. Eine differenzierende Betrachtung unter Berücksichtigung des sozioökonomischen Status. Dissertation an der Universität Bremen 2012

21 Gesundheitsamt Bremen. Was fördert, was behindert das Stillen? Ergebnisse der Bremer Stillstudie.Vortrag zur 39. Sitzung der NSK in Berlin, 15.04.2013 http://www.gesundheitsamt.bremen.de/sixcms/ media.php/13/4_Stillstudie\%20Bremen2013.pdf

22 Bayerisches Landesamt für Gesundheit und Lebensmittelsicherheit. STELLA-Studie zum Ernährungsverhalten im Säuglingsalter Interventionsprojekt zur Verbesserung der Stillbedingungen in einer Modellregion. Abschlussbericht 2011

23 Schoetzau A, Filipiak-Pittroff B, Franke $K$ et al. Effect of exclusive breast-feeding and early solid food avoidance on the incidence of atopic dermatitis in high-risk infants at 1 year of age. Pediatr Allergy Immunol 2002; 13: 234-242

24 Laubereau B, Brockow I, Zirngibl A et al. Effect of breast-feeding on the development of atopic dermatitis during the first 3 years of life results from the GINI-birth cohort study. J Pediatr 2004; 144: 602-607

25 Libuda L, Stimming $M$, Mesch $C$ et al. Frequencies and demographic determinants of breastfeeding and DHA supplementation in a nationwide sample of mothers in Germany. Eur J Nutr 2014; 53: 1335-1344

26 Langnäse K, Mast M, Danielzik S et al. Socioeconomic gradients in body weight of German children reverse direction between the ages of 2 and 6 years. J Nutr 2003; 133: 789-796

27 Landesamt für Verbraucherschutz Sachsen-Anhalt.. Auswirkungen der Umwelt auf die Gesundheit von Kindern. Schulanfängerstudie Sachsen-Anhalt 1991-2014. Mai 2014; http://www.verbraucherschutz. sachsen-anhalt.de/fileadmin/Bibliothek/Politik_und_Verwaltung/MS/ Verbraucherschutz/publikationen/fb2/schulanfaengerstudie_inter net.pdf

28 Lange C, Schenk L, Bergmann $R$. Verbreitung, Dauer und zeitlicher Trend des Stillens in Deutschland. Ergebnisse des Kinder- und Jugendgesundheitssurveys (KIGGS). Bundesgesundheitsblatt Gesundheitsforschung Gesundheitsschutz 2007; 50: 624-633

29 von der Lippe E, Brettschneider AK, Gutsche J et al. Factors influencing the prevalence and duration of breastfeeding in Germany: Results of the KiGGS study: first follow up (KiGGS Wave 1). Bundesgesundheitsblatt Gesundheitsforschung Gesundheitsschutz 2014; 57: 849-859

30 Foterek K, Hilbig A, Alexy U. Breastfeeding and weaning practices in the DONALD study. Age and time trends. J Pediatr Gastroenterol Nutr 2014; 58: 361-367

31 Hilbig A, Kersting $M$. Effects of age and time on energy and macronutrient intake in German infants and young children: results of the DONALD study. J Pediatr Gastroenterol Nutr 2006; 43: 518-524

32 Danielzik S, Czerwinski-Mast M, Langnäse K et al. Parental overweight, socioeconomic status and high birth weight are the major determinants of overweight and obesity in 5-7 y-old children: baseline data of the Kiel Obesity Prevention Study (KOPS). Int J Obes Relat Metab Disord 2004; 28: 1494-1502

33 Hunsberger $M$, Lanfer $A$, Reeske $A$ et al. Infant feeding practices and prevalence of obesity in eight European countries - the IDEFICS study. Infant feeding practices and prevalence of obesity in eight European countries - the IDEFICS study. Public Health Nutr 2013; 16: 219-227

34 von Kries $R$, Koletzko B, Sauerwald $T$ et al. Breast feeding and obesity: cross sectional study. BMJ 1999; 319: 147-150

35 Kalies $H$, Heinrich J, Borte $N$ et al. The effect of breastfeeding on weight gain in infants: results of a birth cohort study. Eur J Med Res 2005; 10: $36-42$

36 Rothenbacher $D$, Bode $G$, Brenner $H$. History of breastfeeding and Helicobacter pylori infection in preschool children: results of a population-based study from Germany. Int J Epidemiol 2002; 31: 632-637

37 Rothenbacher $D$, Weyermann $M$, Beermann $C$ et al. Breastfeeding, soluble CD14 concentration in breast milk and risk of atopic dermatitis and asthma in early childhood: birth cohort study. Clin Exp Allergy 2005; 35: 1014-1021 
38 Weyermann $M$, Rothenbacher $D$, Brenner $H$. Duration of breastfeeding and risk of over-weight in childhood: a prospective birth cohort study from Germany. Int J Obes (Lond) 2006; 30: 1281-1287

39 Hummel S, Pflüger M, Kreichauf S et al. Predictors of overweight during childhood in offspring of parents with type 1 diabetes. Diabetes Care 2009; 32: 921-925

40 Rapp K, Schick KH, Bode H et al. Type of kindergarten and other potential determinants of overweight in pre-school children. Public Health Nutr 2005; 8: 642-949

41 Gillespie B, d'Arcy $H$, Schwartz $K$ et al. Recall of age of weaning and other breastfeeding variables. Int Breastfeed J 2006; 1: 4

42 Vobecky JS, Vobecky J, Froda S. The reliability of the maternal memory in a retrospective assessment of nutritional status. J Clin Epidemiol 1988; 41: 261-265

$43 \mathrm{Li} \mathrm{R}$, Scanlon KS, Serdula MK. The validity and reliability of maternal recall of breastfeeding practice. Nutr Rev 2005; 63: 103-110
44 Promislow JH, Gladen BC, Sandler DP. Maternal recall of breastfeeding duration by elderly women. Am J Epidemiol 2005; 161: 289-296

45 Agampodi SB, Fernando S, Dharmaratne SD et al. Duration of exclusive breastfeeding; validity of retrospective assessment at nine months of age. BMC Pediatr 2011; 11: 80

46 Meyer N, Spegel H, Hendrowarsito L et al. Breast-feeding training programme as intervention approach at the hospital level: results of evaluation of the first phase of the STELLA study. Gesundheitswesen 2012; 74: 34-41

47 EU Project on Promotion of Breastfeeding in Europe. Protection, promotion and support of breastfeeding in Europe: a blueprint for action (revised). Luxembourg: European Commission, Directorate Public Health and Risk Assessment; 2008

48 Yngve A, Sjöström M. Breastfeeding in countries of the European Union and EFTA: current and proposed recommendations, rationale, prevalence, duration and trends. Public Health Nutr 2001; 4: 631-645 